\title{
Author Correction: Preoperative prognostic nutritional index is a powerful predictor of prognosis in patients with stage III ovarian cancer
}

\author{
Weiwei Zhang ${ }^{1,2}$, Bin Ye' ${ }^{2}$, Weijiang Liang ${ }^{1}$ \& Yazhou Ren ${ }^{3}$ \\ Correction to: Scientific Reports https://doi.org/10.1038/s41598-017-10328-8, published online 25 August 2017 \\ In the original version of this Article, Affiliations 1, 2 and 3 were not listed in the correct order. The correct affil- \\ iations are listed below:
}

Affiliation 1:

Department of Medical Oncology, Nanfang Hospital, Southern Medical University, Guangzhou BaiYun Road 1838, 510515, Guangzhou, China

Affiliation 2:

Department of Medical Oncology, The Sixth People’s Hospital of Chengdu, 610051, Chengdu, China

Affiliation 3:

Big Data Research Center, School of Computer Science and Engineering, University of Electronic Science and Technology of China, 611731, Chengdu, China

Additionally, the original version of this Article omitted an affiliation for Weiwei Zhang. The correct affiliations for Weiwei Zhang are listed below:

Department of Medical Oncology, Nanfang Hospital, Southern Medical University, Guangzhou BaiYun Road 1838, 510515, Guangzhou, China

Department of Medical Oncology, The Sixth People’s Hospital of Chengdu, 610051, Chengdu, China

Finally, in the original version of this Article, Weiwei Zhang was incorrectly listed as the corresponding author. The correct corresponding authors for this Article are Weijiang Liang and Yazhou Ren. Correspondence and requests for materials should be addressed to W.L. (email: wjliang22@126.com) or Y.R. (email: Yazhou.ren@uestc. edu.cn).

These errors have now been corrected in the PDF and HTML versions of the Article.

${ }^{1}$ Department of Medical Oncology, Nanfang Hospital, Southern Medical University, Guangzhou BaiYun Road 1838, 510515, Guangzhou, China. 'Department of Medical Oncology, The Sixth People's Hospital of Chengdu, 610051, Chengdu, China. ${ }^{3}$ Big Data Research Center, School of Computer Science and Engineering, University of Electronic Science and Technology of China, 611731, Chengdu, China. Correspondence and requests for materials should be addressed to W.L. (email: wjliang22@126.com) orY.R. (email:Yazhou.ren@uestc.edu.cn) 
(i) Open Access This article is licensed under a Creative Commons Attribution 4.0 International License, which permits use, sharing, adaptation, distribution and reproduction in any medium or format, as long as you give appropriate credit to the original author(s) and the source, provide a link to the Creative Commons license, and indicate if changes were made. The images or other third party material in this article are included in the article's Creative Commons license, unless indicated otherwise in a credit line to the material. If material is not included in the article's Creative Commons license and your intended use is not permitted by statutory regulation or exceeds the permitted use, you will need to obtain permission directly from the copyright holder. To view a copy of this license, visit http://creativecommons.org/licenses/by/4.0/.

(C) The Author(s) 2018 